\title{
Growth hormone replacement therapy regulates microRNA-29a and targets involved in insulin resistance
}

\author{
Artur Galimov $^{1,7} \cdot$ Angelika Hartung $^{1} \cdot$ Roman Trepp $^{2} \cdot$ Alexander Mader $^{3}$ • \\ Martin Flück $^{4,8}$ • Axel Linke ${ }^{5}$ Matthias Blüher ${ }^{6}$ Emanuel Christ ${ }^{2} \cdot$ Jan Krützfeldt $^{1,7,8}$
}

Received: 7 March 2015 / Revised: 5 June 2015 / Accepted: 7 July 2015 / Published online: 23 July 2015

(C) Springer-Verlag Berlin Heidelberg 2015

\begin{abstract}
Replacement of growth hormone $(\mathrm{GH})$ in patients suffering from GH deficiency (GHD) offers clinical benefits on body composition, exercise capacity, and skeletal integrity. However, GH replacement therapy (GHRT) is also associated with insulin resistance, but the mechanisms are incompletely understood. We demonstrate that in GH-deficient mice (growth hormone-releasing hormone receptor $(\mathrm{Ghrhr})^{\text {lit/lit}}$ ), insulin resistance after GHRT involves the upregulation of the extracellular matrix (ECM) and the downregulation of microRNA miR-29a in skeletal muscle. Based on RNA
\end{abstract}

Electronic supplementary material The online version of this article (doi:10.1007/s00109-015-1322-y) contains supplementary material, which is available to authorized users.

Jan Krützfeldt

jan.kruetzfeldt@usz.ch

1 Division of Endocrinology, Diabetes, and Clinical Nutrition, University of Zurich and University Hospital, Rämistrasse 100, 8091 Zurich, Switzerland

2 Division of Endocrinology, Diabetes and Clinical Nutrition, University Hospital Bern Inselspital, Bern, Switzerland

3 Division of Trauma Surgery, University Hospital, Zurich, Switzerland

4 Department of Orthopedics, University Hospital Balgrist, Zurich, Switzerland

5 Heart Center Leipzig, University of Leipzig, Leipzig, Germany

6 Department of Medicine, University of Leipzig, Leipzig, Germany

7 Competence Center Personalized Medicine, ETH Zurich and University of Zurich, Zurich, Switzerland

8 Zurich Center for Integrative Human Physiology, University of Zurich, Zurich, Switzerland deep sequencing of skeletal muscle from GH-treated Ghrhr ${ }^{\text {lit/lit }}$ mice, we identified several upregulated genes as predicted miR-29a targets that are negative regulators of insulin signaling or profibrotic/proinflammatory components of the ECM. Using gain- and loss-of-function studies, five of these genes were confirmed as endogenous targets of miR-29a in human myotubes (PTEN, COL3A1, FSTL1, SERPINH1, SPARC). In addition, in human myotubes, IGF1, but not GH, downregulated miR-29a expression and upregulated COL3A1. These results were confirmed in a group of GH-deficient patients after 4 months of GHRT. Serum IGF1 increased, skeletal muscle miR-29a decreased, and miR-29a targets were upregulated in patients with a reduced insulin response (homeostatic model assessment of insulin resistance (HOMA-IR)) after GHRT. We conclude that miR-29a could contribute to the metabolic response of muscle tissue to GHRT by regulating ECM components and PTEN. miR-29a and its targets might be valuable biomarkers for muscle metabolism following $\mathrm{GH}$ replacement.

\section{Key messages}

- GHRT most significantly affects the ECM cluster in skeletal muscle from mice.

- GHRT downregulates miR-29a and upregulates miR-29a targets in skeletal muscle from mice.

- PTEN, COL3A1, FSTL1, SERPINH1, and SPARC are endogenous miR-29a targets in human myotubes.

- IGF1 decreases miR-29a levels in human myotubes.

- miR-29a and its targets are regulated during GHRT in skeletal muscle from humans.

Keywords microRNA · GH · IGF1 · Skeletal muscle · Insulin resistance 
Growth hormone $(\mathrm{GH})$ is an anabolic hormone that increases skeletal muscle mass and bone density [1]. GH action involves, however, also a catabolic effect on fat mass through the upregulation of lipolysis, mainly at the level of the visceral fat depot [2]. These opposing roles of GH might serve as an important mechanism to prevent muscle and bone catabolism during periods of limited caloric intake. The regulation of glucose homeostasis by GH is also determined by GH effects on hepatocytes leading to elevated circulating IGF1 levels and increased glycogenolysis [3] and gluconeogenesis [4]. These complex metabolic effects of GH impose challenges on controlling cardiovascular risk factors in patients suffering from adult-onset GH deficiency (GHD). Patients with GHD have increased frequency of adverse metabolic features such as increased abdominal obesity, insulin resistance, and dyslipidemia [5]. Although short-term GH replacement therapy (GHRT) improves some of these risk factors such as visceral fat mass and hypercholesterolemia, it can also aggravate insulin resistance [6]. Long-term GHRT over 10 years was not able to prevent an increase in the prevalence of the metabolic syndrome compared to baseline, despite improvements of LDL cholesterol [7], and discontinuing long-term GHRT was shown to lead to improved insulin sensitivity [8]. A more detailed understanding of the mechanisms that contribute to altered glucose homeostasis during GHRT is needed to prevent adverse metabolic events and to optimize cardiovascular risk factors during GHRT, for example, by tailoring lifestyle interventions or adjunct pharmacological protocols. Skeletal muscle is an important target tissue for such interventions since it is the major contributor to postprandial insulin-dependent glucose disposal. Moreover, a decreased response to insulin in muscle tissue is frequently observed in humans during GHRT [9].

MicroRNAs (miRNAs) are a family of non-coding RNAs of only 20-24 nucleotides in length. miRNAs have several characteristics which make them important tools for the identification of disease mechanisms. A single miRNA can coordinately regulate the expression of multiple genes involved in different pathways, and therefore, the discovery of altered miRNA expression could unravel gene regulation in complex diseases. Furthermore, miRNAs have remarkable tissue specificity and might be more versatile markers of disease states than protein-coding mRNAs [10]. We therefore hypothesized that miRNA expression in skeletal muscle can help elucidate the mechanisms that contribute to increased insulin resistance after GHRT. Previously, miRNAs have been shown to cause insulin resistance in liver and fat tissue as demonstrated by global miRNA knockout models [11] or pharmacological miRNA inhibitors [12]. Accordingly, the search for miRNAs that are regulated in insulin-resistant states could also provide novel strategies to treat cardiovascular risk factors during GHRT.
Employing an animal model of GHD allowed us to identify the regulation of miR-29a and several of its targets during GHRT and to provide novel insights into the regulation of muscle metabolism during GHRT in both animals and humans.

\section{Research design and methods}

\section{Participants}

Muscle biopsies from 10 patients with adult-onset GHD for at least 12 months (three women, seven men, and age range 24 to 62 years) were recruited from a previously reported trial at the University of Bern, Switzerland [13]. Three patients had craniopharyngiomas, three patients had hormone-inactive pituitary adenomas, one patient had a Rathke's cleft cyst, one patient had traumatic hypopituitarism, one patient had a macroprolactinoma, and one patient had hypophysitis. Inclusion and exclusion criteria have been described in detail previously [13]. Biopsies were harvested from the middle portion of the right tibialis anterior muscle under local anesthesia before and after a total of 4 months of GHRT [13]. Homeostatic model assessment of insulin resistance (HOMA-IR) was calculated as fasted insulin $(\mu \mathrm{U} / \mathrm{ml}) \times$ fasted glucose $(\mathrm{mmol} / \mathrm{l}) /$ 22.5. FSTL1 and SPARC were measured in EDTA plasma obtained in the fasted state after 1:120 dilution in PBS using ELISAs from Uscn Life Science Inc.

A second cohort of participants was recruited from the University of Leipzig, Germany [14]. Patients of either sex referred to the University of Leipzig Heart Center, Germany, were invited to participate if they displayed impaired fasting glucose $(>6.0$ and $<7.0 \mathrm{mmol} / 1$ with $\mathrm{HbAlC}<6 \%$ ) or impaired glucose tolerance $(>7.8$ and $<11.1 \mathrm{mmol} / \mathrm{l}) 2 \mathrm{~h}$ after oral intake of $75 \mathrm{~g}$ glucose and angiographic evidence of coronary artery disease $(>50 \%$ stenosis diameter in at least one major epicardial artery). Vastus lateralis muscle biopsies were obtained under local anesthesia and immediately snap frozen in liquid nitrogen. Seventy-five-gram oral glucose tolerance tests (OGTT) were performed under standardized conditions. In total, muscle biopsies from 33 patients $(27$ males, 6 females) were analyzed. We calculated the metabolic clearance rate $(\mathrm{MCR})(19.240-0.281 \times \mathrm{BMI}-0.00498 \times \mathrm{Ins} 120-$ $0.333 \times$ Gluc 120$)$ and the insulin sensitivity index (ISI) $(0.222-0.00333 \times$ BMI $-0.0000779 \times \operatorname{Ins} 120-0.000422 \times$ age) from the OGTT since they correlate well with insulin sensitivity as measured by the glucose clamp technique ( $r$ values of 0.79 for MCR and ISI, respectively [15]). Average age was $62.1 \pm 1.1$ years, BMI $30.4 \pm 0.7 \mathrm{~kg} / \mathrm{m}^{2}$, and glucose during OGTT at $0 \mathrm{~h}$ was $6.1 \pm 0.1 \mathrm{mM}$, at $1 \mathrm{~h} 11.7 \pm 0.4 \mathrm{mM}$, and at $2 \mathrm{~h} 9.1 \pm 0.3 \mathrm{mM}$. All values are means with SEM.

For isolation of muscle cells, human skeletal muscle was obtained from elective surgery of the lower limb performed on 
two healthy donors (males, age 20 and 22 years, BMI $22 \mathrm{~kg} /$ $\mathrm{m}^{2}$ ) at the University Hospital of Zurich.

All study protocols were approved by the local ethics committees. All subjects gave written informed consent before enrollment.

\section{Animals}

All mice were maintained in a 12 -h light/dark cycle in a pathogen-free animal facility. C57BL/6J-growth hormonereleasing hormone receptor $(G h r h r)^{l i t} / \mathrm{J}$ mice were purchased from Jackson Laboratories. Between 10 and 14 weeks of age, mice were injected once daily with recombinant GH subcutaneously (Genotropin, Pfizer) at a dose of $6 \mu \mathrm{g} / \mathrm{g}$ bodyweight per day. We chose this dosage since $6 \mu \mathrm{g} / \mathrm{g}$ bodyweight induced insulin resistance in GHD mice in a previous report [16]. After 21 days, mice were sacrificed and the tibialis anterior and soleus muscle and liver tissue removed, snap frozen in liquid nitrogen, and stored at $-80{ }^{\circ} \mathrm{C}$. Serum was collected from tail-vein blood and analyzed for glucose using FreeStyle Lite (Abbott) and for insulin using the ultrasensitive mouse insulin ELISA kit (Crystal Chem). C57BL6/6J mice were purchased from Harlan Laboratories. Starting at 4 weeks of age, mice were fed a $58 \%$ fat and sucrose diet (D12331; Research Diets, USA). After 20 weeks, mice were sacrificed and the tibialis anterior muscle removed and processed as described above. All animal studies were approved by the ethics committee of the Kantonale Veterinäramt Zürich, and the principles of laboratory animal care were followed.

\section{RNA isolation, $q R T-P C R$, and mRNA sequencing}

Total RNA was isolated from muscle tissues using the TRIzol reagent (Invitrogen). For quantitative reverse transcription (qRT)-PCR, total RNA was subjected to DNAse digestion followed by complementary DNA preparation with random hexamer primers using Super Script III Reverse Transcriptase (Invitrogen). Messenger RNA (mRNA) was analyzed by quantitative real-time PCR using the FastStart Universal SYBR Green Master Mix (Roche) with a 7500 FastStart Real-Time PCR system (Applied Biosystems). Transcript levels were normalized to $18 \mathrm{~S}$ ribosomal RNA. Primer sequences are provided in ESM 1: Table S6. miRNA levels were measured using qRT-PCR with the TaqMan miRNA assays (Applied Biosystems). The ubiquitous miR-let-7 was used as an endogenous control based on its stable expression in all samples and its conservation between mice and humans. For mRNA and small RNA profiling, RNA was subjected to Illumina deep sequencing using a service from LC Sciences (Houston, TX, USA). The sequence results were obtained as FPKM (fragment per kilobase of exons per million reads) for each transcript.
Primary human myotube cultures, miRNA transfection, and western blotting

Human skeletal muscle tissue was subjected to collagen digestion, and myogenic progenitor cells were isolated using flow cytometry based on CD56 expression and the absence of CD15, CD31, and CD45 staining. Cells were grown on collagen-coated plates in culture medium $(1: 1 v / v$ F10 nutrient mixture and Dulbecco's modified Eagle's medium (DMEM) low glucose containing $20 \%$ fetal bovine serum (FBS), $1 \%$ penicillin/streptomycin (P/S), and $5 \mathrm{ng} / \mathrm{ml}$ basic FGF). Experiments were always performed on two independent primary muscle cell cultures obtained from the two healthy donors and analyzed in different passages (not higher than passage 10), e.g., $n=4$, indicating two different passages each from the two independent primary cultures. Differentiation was initiated when myoblasts reached subconfluency by changing the media to DMEM containing $2 \%$ horse serum and $1 \% \mathrm{P} / \mathrm{S}$. IGF1 was measured in the supernatant using an ELISA for human IGF1 from Abcam. miRNA transfection was performed on human myotubes at day 4 of differentiation using Lipofectamine RNAimax (Invitrogen) and human miRNA mimics (38 nM, Mission; Sigma-Aldrich) or miRNA inhibitors (12 nM, antagomirs [17]). Concentrations of oligonucleotides were chosen based on dose-response curves and miR-29a target gene expression in human myotubes (data not shown). Scrambled mimics or antagomirs were used as controls. Cells were harvested for RNA isolation 24 or $48 \mathrm{~h}$ after transfection using the TRIzol reagent (Invitrogen). Myotubes were incubated for the indicated time points with IGF1 or GH. We chose $1 \mu \mathrm{g} / \mathrm{ml} \mathrm{GH}$ according to previous reports that demonstrated $500-1000 \mathrm{ng} / \mathrm{ml} \mathrm{GH}$ to have maximal effects on GH signaling in myoblasts and myotubes [18-20]. For IGF1 incubations, we chose $76 \mathrm{ng} / \mathrm{ml}(10 \mathrm{nM})$, a concentration for which Baudry et al. [21] demonstrated maximal effects on glucose uptake and IGF1 signaling in myotubes. For western blotting, myotubes were lysed in RIPA buffer and separated by SDSPAGE, transferred to PVDF membranes, and primary antibodies were used to detect myosin heavy chain (MHC, MF-20, DSHB), GAPDH (Proteintech, \#10494), AKT, and phosphoAKT (Cell Signalling, \#2920, \#4058).

\section{Statistical analyses}

Data shown as arbitrary units (AU) are normalized to the average value of the control group. Groups were compared using Student's $t$ test in Excel software. Correlation and ANOVA analysis was performed using the GraphPad Prism software. Fold-change regulations were analyzed using onesample $t$ test with the hypothetical means of 1 using the GraphPad Prism software. $P$ values smaller than 0.05 were considered significant. Results are shown as means \pm SEM. 


\section{Results}

To elucidate the mechanisms that regulate muscle metabolism during GHRT, we chose Ghrhr ${ }^{\text {lit } / i t}$ mice as a model for GHD. $G h r h r^{\text {lit/lit }}$ mice are homozygous for a missense mutation in the growth hormone-releasing hormone receptor (Ghrhr) resulting in pituitary and serum Gh levels of less than $5 \%$ and serum IGF1 levels of 15-20\% of normal [22]. As expected from previous protocols [16], 3 weeks of GHRT induced insulin resistance in the Gh-deficient mice compared to saline injections as demonstrated by increased fasting and random glucose values and higher insulin levels (Fig. 1a). GHRT also increased bodyweight (data not shown). To identify relevant changes in gene expression that could affect insulin sensitivity in skeletal muscle, we performed next-generation sequencing on total RNA isolated from muscle tissue from GHD mice with or without GHRT. The GH treatment significantly regulated 1422 transcripts (1063 upregulated and 360 downregulated, ESM 1: Table S1). Strikingly, the three most significantly induced functional clusters in the GHRT group involved extracellular matrix (ECM) organization, while the three functional clusters with the most significant downregulation included fatty acid metabolism (Fig. 1b). To identify novel mechanisms for the regulation of ECM during GHRT, we turned to the miR-29 microRNA family (miR-29a, miR-29b, and miR-29c) which is a well-known regulator of ECM [23]. Small RNA sequencing identified miR-29a as the most abundant member of the miR-29 family in skeletal muscle from mice and humans and in muscle groups with either predominant fiber type I (M. soleus) or fiber type II (M. tibialis anterior, M. fibularis brevis, M. rectus femoris) (Fig. 1c). Importantly, miR-29a was significantly decreased in our cohort of a

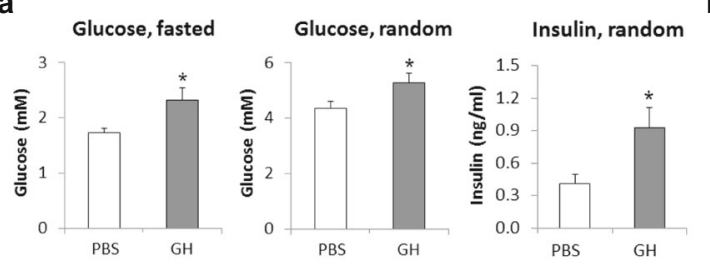

b

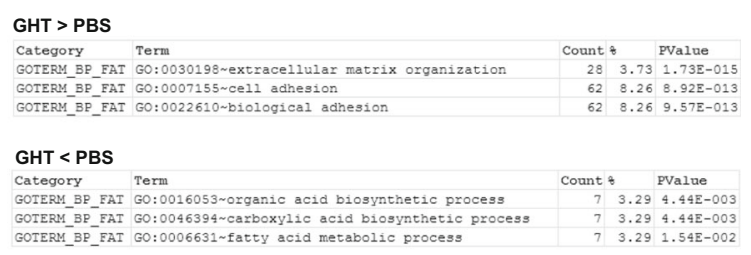

C
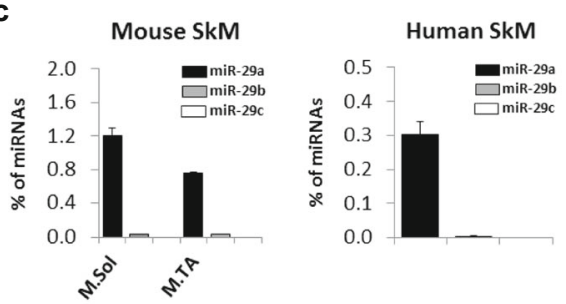

e

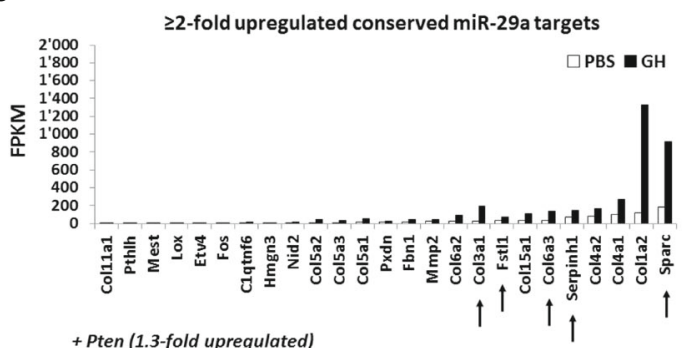

Fig. 1 GHRT regulates miR-29a expression in skeletal muscle from GHD mice. GHD mice were treated with PBS or GH for 3 weeks and serum samples and tibialis anterior and soleus muscle harvested. a Blood glucose and serum insulin levels in GHD mice with $(\mathrm{GH})$ or without (PBS) 3 weeks of GHRT. b Total RNA isolated from tibialis anterior muscle from GHD mice treated with PBS or GH was analyzed using RNA deep sequencing. The three most significantly regulated functional clusters are depicted for the group of genes that are increased after GHRT (GHT $>$ PBS) or decreased (GHT $<$ PBS). $\mathbf{c}$ RNA from mouse soleus muscle (M.Sol), tibialis anterior muscle (M.TA), or lower leg muscles from two healthy human subjects (M. rectus femoris and M. fibularis brevis, respectively) was analyzed using small RNA deep sequencing and results expressed as percent of all detected miRNA sequences. $n=2$ for
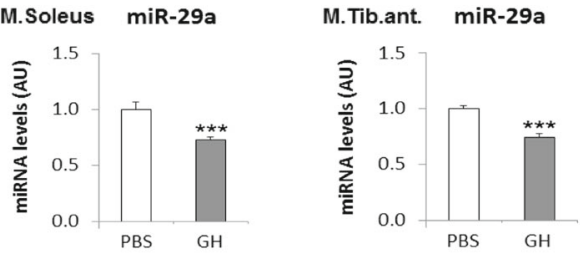

f miR-29a targets

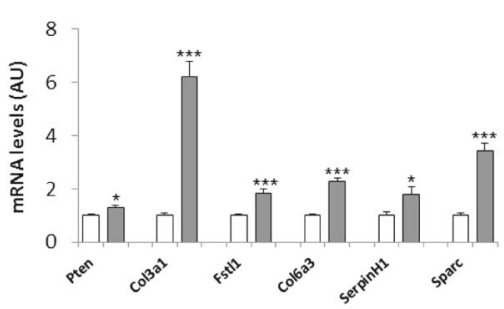

each muscle group. $\mathbf{d}$ qRT-PCR analysis of miR-29a in RNA from tibialis anterior muscle normalized to miR-let-7 and shown as arbitrary units (AU). Analysis was performed in the same mice as described in a. e All predicted miR-29a targets that are conserved between mice and humans and upregulated by GH in the RNA sequencing data described in $\mathbf{b} \geq 2$ fold shown as FPKM (fragments per kilobase of exons per million fragments mapped). Selected targets as described in the text are pointed out by arrows. f qRT-PCR analysis of gene expression in skeletal muscle after GH (gray) or PBS treatment (white). mRNA was normalized to 18S RNA and shown as AU. Results $(\mathbf{a}, \mathbf{d}, \mathbf{f})$ show combined data for male and female mice ( $n=7$ for GH and $n=8$ for PBS) and were analyzed using Student's $t$ test. $* p<0.05, * * * p<0.001$ 
GHRT treated mice in both type I (M. soleus) and type II (M. tibialis anterior) muscle types (Fig. 1d). We then searched for all predicted miR-29a targets in the group of upregulated genes from our sequencing data that are conserved between mice and humans using the TargetScan program [24] (ESM 1: Table S2). Importantly, the list of predicted targets that were $\geq 2$-fold upregulated was enriched for profibrotic and proinflammatory genes that are part of the ECM (Fig. 1e and ESM 1: Table S3), and we chose five candidates from the 10 highest expressed genes (COL3A1, FSTL1, COL6A3, SERPINH1, SPARC). We selected COL3A1 and COL6A3 from the seven collagen isoforms among these genes, because their upregulation has consistently been shown to correlate with skeletal muscle insulin resistance in humans $[25,26]$. From the list of miR-29a targets that were upregulated less than twofold, we selected PTEN since it is a well-established inhibitor of the intracellular insulin signaling [27]. The regulation of these selected predicted miR-29a targets during GHRT was confirmed using qRT-PCR (Fig. 1f). Together, these data identify miR-29a and a set of its targets that could contribute to the changes in glucose tolerance during GHRT.

To test whether the confirmed targets of miR-29a could also be relevant for human skeletal muscle, we transfected miR-29a mimics or inhibitors (antagomirs) into human myotubes from two healthy donors. A significant repression or induction of miRNA targets at the mRNA level was reached after $48 \mathrm{~h}$ of mimic or inhibitor (antagomirs) transfection, respectively, with a substantial decrease of mRNA repression already observed after $24 \mathrm{~h}$ (Fig. 2). As expected, the effect of miR-29a inhibition was in general weaker than that of the overexpression, given that endogenous miR-29a levels are lower than the supra-physiological overexpression with the mimics. Overexpression or inhibition of miR-29a did not change morphology or differentiation state of the myotubes as evaluated by light microscopy (data not shown) and expression of MHC protein (ESM 1: Fig. S1). Also, insulin-stimulated AKT phosphorylation was unaltered indicating that miR-29a targets do not induce insulin resistance in human muscle cells in a cell autonomous manner (ESM 1: Fig. S1). Overall, our approach validated five of the six predicted miR-29a targets as endogenous targets in differentiated human skeletal muscle cells (PTEN, COL3A1, FSTL1, SERPINH1, SPARC).

To test whether the regulation of miR-29a in skeletal muscle was directly related to the GHRT, we incubated human myotubes with either GH or IGF1. Liver is the major source of GH-stimulated circulating IGF1 levels [28], but GH also stimulates IGF1 mRNA levels in extrahepatic tissues such as skeletal muscle [29]. However, we did not detect significant IGF1 levels in the supernatant from human myotubes upon GH incubation (data not shown), indicating that the effects of GH and IGF1 can be addressed separately in these cells. We found that IGF1, but not GH, gradually decreased miR-29a levels over the course of $48 \mathrm{~h}$ (Fig. 3a). Furthermore, COL3A1 expression was reversely correlated to miR-29a levels (Fig. 3b). Other confirmed miR-29a targets were not significantly regulated (data not shown) and might need longer incubation times or a more pronounced downregulation of miR-
Fig. 2 Target gene regulation in human myotubes by miR-29a. Human myotubes were transfected with miR-29a mimics or antagomirs or the respective scrambled controls. After the indicated time intervals, cells were harvested for RNA isolation. Results are shown for qRT-PCR analysis after miR-29a mimic transfection (gray) compared to control (white), $n=4$, or after antagomir-29a transfection (gray) compared to control (white), $n=$ 4-5. Results were normalized to 18S RNA and shown as arbitrary units (AU). Student's $t$ test was used to compare the two groups. ${ }^{*} p<0.05,{ }^{* *} p<0.01,{ }^{* * *} p<0.001$
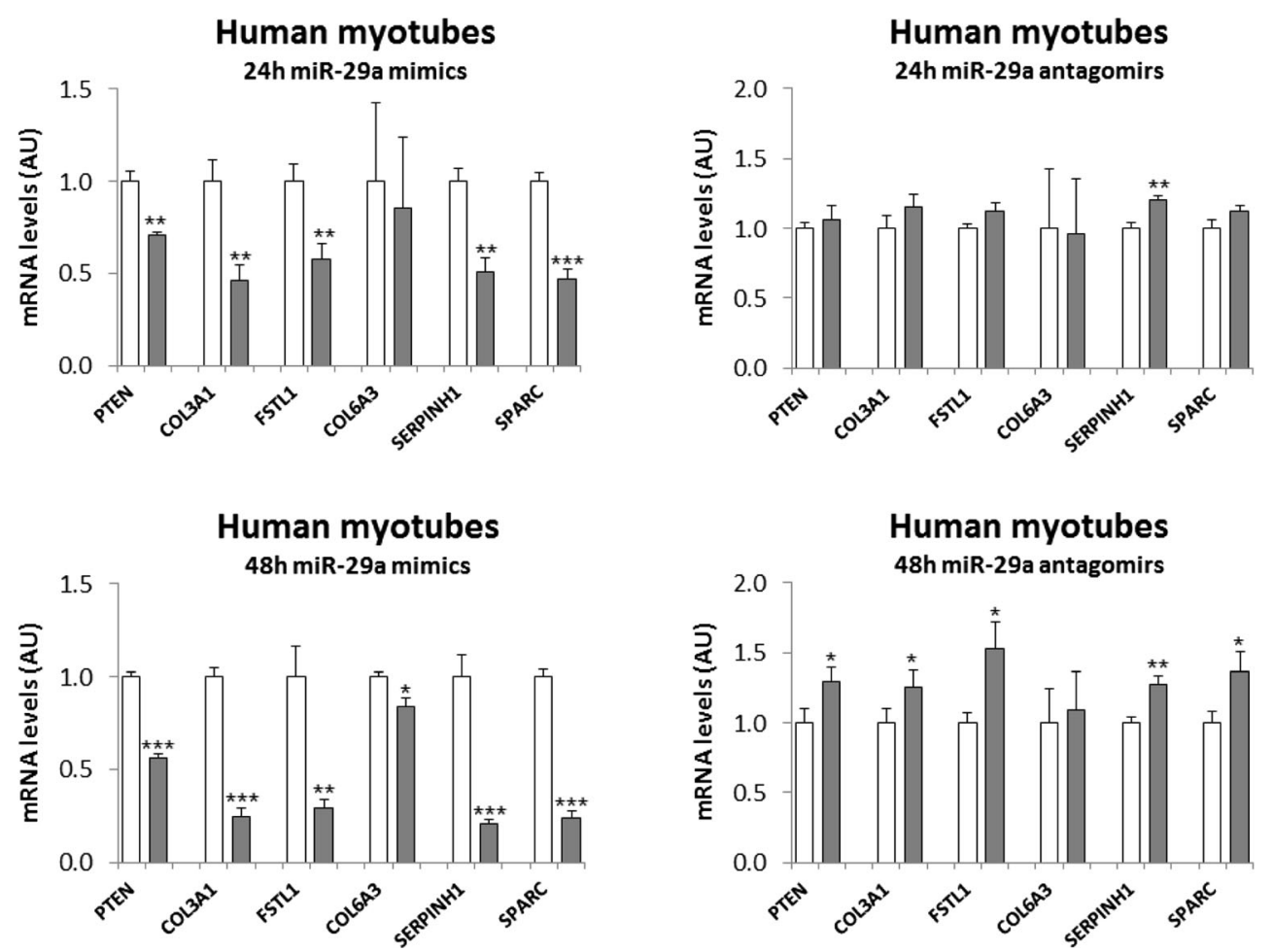

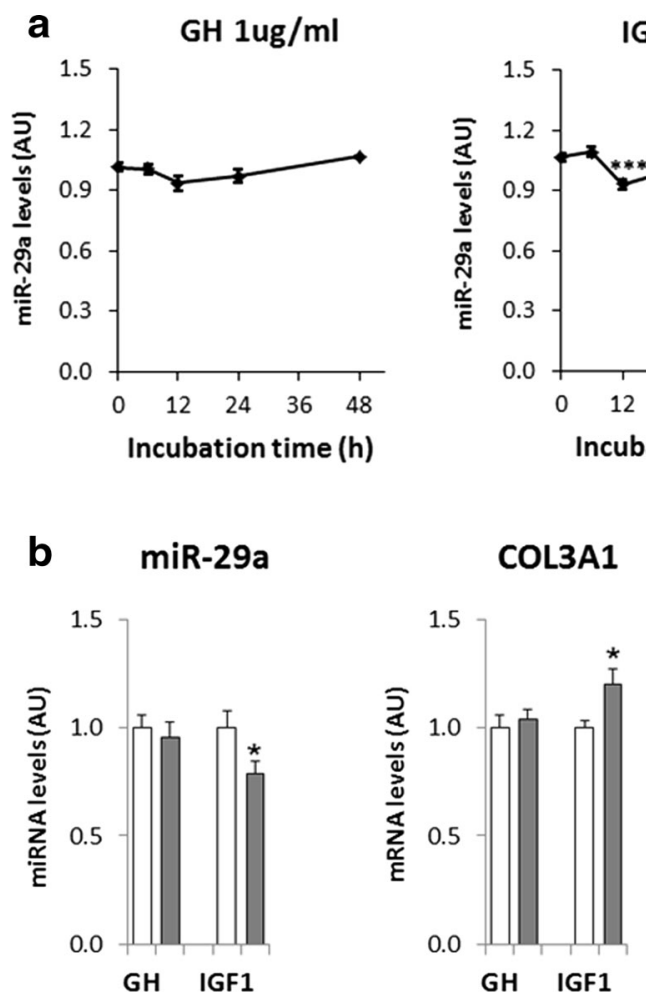

Fig. 3 IGF1, but not GH, downregulates miR-29a levels in human myotubes. a Human myotubes were incubated for the indicated time intervals with $1 \mu \mathrm{g} / \mathrm{ml} \mathrm{GH}$ or $76 \mathrm{ng} / \mathrm{ml}(10 \mathrm{nM}) \mathrm{IGF} 1$ before RNA was isolated. miR-29a levels were analyzed using qRT-PCR and normalized to miR-let-7. $n=4$ for GH and $n=10$ for IGF1. b Direct comparison of the effects of GH and IGF1 on miR-29a levels and COL3A1 expression after 48-h incubation in an independent set of experiments, $n=3-5$. Results are shown as arbitrary units (AU). Changes in a were analyzed using ANOVA and Dunnett's post test comparing all samples to the control samples (at $0 \mathrm{~h}$ ). The effects of GH and IGF1 on miR-29a and COL3A1 levels (b) were analyzed using Student's $t$ test. ${ }^{*} p<0.05,{ }^{* * *} p<0.001$

$29 \mathrm{a}$ as observed for the adult skeletal muscle in vivo. We conclude that downregulation of miR-29a in skeletal muscle during GHRT could be a direct consequence of increased IGF1 levels.

To understand whether the regulation of miR-29a and its targets is important for the change in insulin sensitivity during GHRT in humans, we studied a cohort of GHD patients that received GHRT over 4 months. We used HOMA-IR to group the patients with an increase or decrease in insulin sensitivity (IS) after GHRT. As expected, insulin levels negatively correlated to IS in that patients with decreased IS also had significantly higher insulin levels (increased HOMA-IR) (Table 1). Furthermore, triglyceride levels were significantly higher in the insulin-resistant group after GHRT compared to patients with improved insulin sensitivity. We further analyzed the impact of GHRT on IGF1 and miR-29a levels compared to the baseline levels of the participants. GHRT significantly induced serum IGF1 levels and decreased miR-29a expression in skeletal muscle (Fig. 4a). Importantly, a significant induction of IGF1 and downregulation of miR-29a was observed in the subgroup of patients with increased HOMA-IR, but not with decreased HOMA-IR (Fig. 4a). In addition, we found a striking correlation between the decrease of miR-29a levels and the change of insulin sensitivity during GHRT (Fig. 4b). We conclude that downregulation of miR-29a in skeletal muscle after GHRT is related to the increase in serum IGF1 and correlates with the change in insulin sensitivity.

Next, we analyzed the five validated miR-29a targets in the GHRT human cohort. We observed a significant induction of these target genes after GHRT confirming a conserved role of these genes in our animal model of GHRT and the patients (Fig. 5a). To assess the contribution of miR-29a to the regulation of these genes, we separately analyzed their regulation in the subgroups with improved insulin sensitivity (HOMAIR decreased) or decreased insulin sensitivity (HOMA-IR increased). Importantly, a significant miR-29a target upregulation was only observed in subjects who developed insulin resistance (Fig. 5a), consistent with the significant downregulation of miR-29a in this group. We conclude that miR-29a could contribute to the effects of GHRT on skeletal muscle insulin resistance by participating in the regulation of intracellular insulin signaling and remodeling of the ECM (Fig. 5b).

To test whether regulation of miR-29a in skeletal muscle also occurs in insulin resistance associated with obesity, we investigated muscle biopsies from both a cohort of prediabetic patients with abnormal OGTT and an average BMI of 30.4 \pm $0.7 \mathrm{~kg} / \mathrm{m}^{2}$ as well as in mice fed a high-fat diet (HFD). However, miR-29a did not correlate with the metabolic clearance rate (MCR) or insulin sensitivity index (ISI) in the 33 insulinresistant subjects (ESM 1: Fig. S2). In mice, HFD led to an increase in fasted and random blood glucose, but miR-29a levels were not altered in skeletal muscle (ESM 1: Fig. S3). We also did not detect any differences in miR-29a target gene expression in skeletal muscle from HFD mice (ESM 1: Fig. S3). These data show that miRNA regulation and gene expression in skeletal muscle after GHRT differs from obesity-associated insulin resistance.

\section{Discussion}

This is the first study to report altered miRNA expression during GHRT. Using next-generation sequencing, we identify the ECM cluster as the most significant upregulated group of genes in skeletal muscle following GH treatment of Ghrhr $r^{\text {lit/it }}$ mice. Next, by performing small RNA sequencing, we demonstrate that miR-29a is the most abundant member of the miR-29 family in skeletal muscle and that its expression is decreased during GHRT. Furthermore, we validate five genes as targets of miR-29a in both mice and human myotubes. One of these genes, PTEN, is a known suppressor of insulin signaling while the remaining four genes are all involved in ECM remodeling. These results are corroborated in human patients 
Table 1 Characteristics of the GHD patients at baseline and after 4 months of GHRT

\begin{tabular}{|c|c|c|c|c|c|c|c|}
\hline & \multicolumn{2}{|l|}{ IS increased } & \multirow{2}{*}{$\begin{array}{l}p \text { values } \\
\text { Start vs. } \\
4 \text { months }\end{array}$} & \multicolumn{2}{|l|}{ IS decreased } & \multirow{2}{*}{$\begin{array}{l}p \text { values } \\
\text { Start vs. } \\
4 \text { months }\end{array}$} & \multirow{2}{*}{$\begin{array}{l}p \text { values } \\
\text { IS decreased vs. } \\
\text { increased } 4 \text { months }\end{array}$} \\
\hline & GHRT start & GHRT 4 months & & GHRT start & GHRT 4 months & & \\
\hline $\operatorname{Sex}(n)$ & 5 & & & 5 & & & \\
\hline Male & 4 & & & 3 & & & \\
\hline Female & 1 & & & 2 & & & \\
\hline Age (years) & $35.0 \pm 3.7$ & & & $47.6 \pm 6.5$ & & & 0.10 \\
\hline BMI $\left(\mathrm{kg} / \mathrm{m}^{2}\right)$ & $27.1 \pm 1.3$ & $27.4 \pm 1.5$ & 0.63 & $25.5 \pm 0.8$ & $25.6 \pm 1.0$ & 0.70 & 0.30 \\
\hline Lean mass (kg) & $55.7 \pm 5.0$ & $57.8 \pm 5.4$ & 0.13 & $56.1 \pm 7.8$ & $60.3 \pm 8.8$ & 0.09 & 0.79 \\
\hline Waist (cm) & $93.8 \pm 3.3$ & $93.1 \pm 3.9$ & 0.53 & $95.9 \pm 4.5$ & $94.0 \pm 4.9$ & 0.29 & 0.88 \\
\hline Fasted glucose (mM) & $4.6 \pm 0.3$ & $4.6 \pm 0.3$ & 0.56 & $4.6 \pm 0.3$ & $5.1 \pm 0.1$ & 0.13 & 0.07 \\
\hline Fasted insulin (mU/l) & $4.8 \pm 1.5$ & $3.0 \pm 1.4$ & 0.01 & $5.0 \pm 1.1$ & $7.8 \pm 1.6$ & 0.02 & 0.03 \\
\hline HOMA-IR & $1.0 \pm 0.4$ & $0.7 \pm 0.4$ & 0.01 & $1.1 \pm 0.3$ & $1.8 \pm 0.3$ & 0.01 & 0.04 \\
\hline IGF1 $(\mu \mathrm{g} / \mathrm{l})$ & $104.8 \pm 20.9$ & $175.2 \pm 23.6$ & 0.06 & $93.0 \pm 24.3$ & $180.4 \pm 29.0$ & 0.01 & 0.88 \\
\hline Triglycerides (mM) & $1.7 \pm 1.0$ & $0.9 \pm 0.3$ & 0.26 & $1.8 \pm 0.2$ & $2.1 \pm 0.3$ & 0.16 & 0.01 \\
\hline NEFA (mM) & $0.5 \pm 0.1$ & $0.6 \pm 0.1$ & 0.78 & $0.7 \pm 0.1$ & $0.7 \pm 0.0$ & 0.58 & 0.12 \\
\hline Total cholesterol (mM) & $4.9 \pm 0.5$ & $4.8 \pm 0.9$ & 0.92 & $5.5 \pm 0.4$ & $5.0 \pm 0.4$ & 0.07 & 0.83 \\
\hline LDL (mM) & $3.4 \pm 0.3$ & $3.3 \pm 0.8$ & 0.86 & $3.9 \pm 0.4$ & $3.4 \pm 0.4$ & 0.01 & 0.85 \\
\hline HDL (mM) & $1.2 \pm 0.1$ & $1.4 \pm 0.1$ & 0.18 & $1.2 \pm 0.1$ & $1.2 \pm 0.1$ & 0.86 & 0.25 \\
\hline
\end{tabular}

Values shown are means \pm SEM

Significant $p$-values $(p<0.05)$ are indicated in italic font style

where again we could demonstrate that miR-29a and its endogenous targets are significantly regulated by GHRT in the group of insulin-resistant patients. We therefore conclude that miR-29a along with its targets could participate in muscle insulin resistance following GHRT.

GH has a profound effect on the ECM and has been shown to induce collagen synthesis in skeletal muscle [30]. The induction of collagens in human skeletal muscle has been associated with insulin resistance $[25,26,31]$, and the increase in ECM and collagens during GHRT might also negatively influence muscle metabolism. Our data support this notion since GHRT in mice caused hyperinsulinemia and induced ECM remodeling in skeletal muscle. In animal models, pharmacological or genetic ECM manipulation alters insulin sensitivity in skeletal muscle $[32,33]$. The accumulation of ECM in skeletal muscle is linked to increased inflammation $[25,26]$ and decreased insulin signaling [31] by the interaction of collagens with integrins in endothelial cells and immune cells leading to decreased muscle vascularization [34] and an increased immune response [35]. miR-29a can further influence the collagen matrix by targeting SERPINH1 (heat shock protein 47), which is important for maturation and secretion of collagens [36], and SPARC (osteonectin, BM-40), which promotes formation of collagen fibrils [37]. FSTL1 and SPARC are also secreted from skeletal muscle into the circulation upon exercise and are considered myokines [38, 39]. Only four myokines are regulated by GHRT according to our RNAseq data (ESM 1: Table S4), and FSTL1 and SPARC show the highest regulation. Importantly, both FSTL1 and SPARC are linked to inflammation and insulin resistance. FSTL1 is a proinflammatory cytokine that is increased in adipose tissue of obese mice and in the serum of overweight and obese subjects [40]. SPARC is a profibrotic protein that is increased in adipose tissue from obese humans [41] and in plasma of insulin-resistant women with gestational diabetes [42]. In our study, we did not observe an induction of plasma FSTL1 or SPARC after GHRT (ESM 1: Fig. S4), which might require stimulation with an acute exercise bout [38, 39]. Interestingly, FSTL1 levels even decreased during GHRT, most pronounced in the subgroup with a decrease in their insulin sensitivity (ESM 1: Fig. S4), which deserves further testing. Increased myokine secretion after exercise bouts during GHRT could also have beneficial effects, since increased SPARC levels after exercise suppressed colon tumorigenesis [38] and muscle-derived FSTL1 attenuated neointimal formation in response to arterial injury [43]. Together, we propose that disinhibition of ECMspecific target genes of miR-29a could contribute to impaired insulin signaling following GH treatment (Fig. 5b).

A possible mechanism by which GH might affect ECM and miR-29a expression is through IGF1. One report links GH treatment to muscle ECM synthesis and increases in IGF1 levels in serum as well as mRNA in skeletal muscle [44]. In our study, not only did IGF1 decrease miR-29a levels in human myotubes but a significant reduction of skeletal 

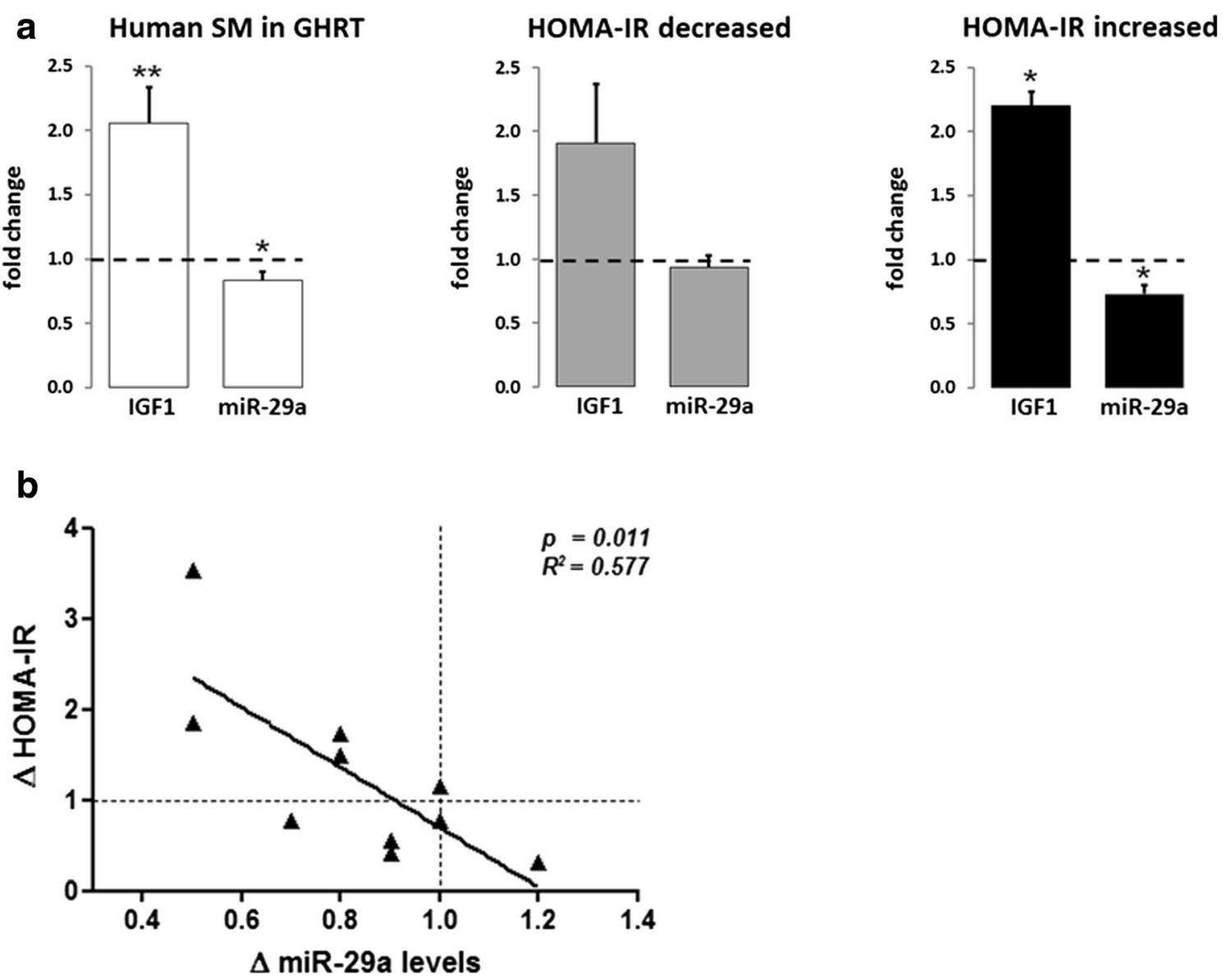

Fig. 4 Increased serum IGF1 and decreased miR-29a levels in skeletal muscle from GHD patients with decreased insulin sensitivity after GHRT. Ten GHD patients were subjected to GHRT for 4 months. a Changes in serum IGF1 and miR-29a levels in biopsies from the tibialis anterior muscle in GHD patients after 4 months of GHRT. Values are shown for the whole cohort, $n=10$, or separately analyzed for individuals with improved IS (HOMA-IR decreased), $n=5$, or worsened IS (HOMA-IR increased), $n=5$, after GHRT. b Relationship between the change in

muscle miR-29a was only observed in the subgroup of insulin-resistant patients with a significant increase in serum IGF1. Our results support the conclusion that GH, through IGF1 signaling, could induce expression of ECM components partly by decreasing the expression of miR-29a. There is currently no evidence to support a major role of $\mathrm{GH}$ on fiber typing [45] and the transcripts of the two major MHC isoforms in tibialis anterior muscle, Myh1 and Myh4, were not regulated after GHRT in our animal model (ESM 1: Table S5).

The contribution of miR-29a to the regulation of its targets during GHRT is best reflected in the divergent regulation of these targets between the group of insulin-resistant patients with decreased miR-29a levels compared to patients with improved insulin sensitivity and no change in miR-29a levels following GHRT. Although the effect of miR-29a on its targets is mild, the target regulation would be expected to occur over a long period of time and thereby slowly contribute to the deterioration of insulin signaling in skeletal muscle. insulin sensitivity ( $\triangle$ HOMA-IR) induced by 4 months of GHRT and the change in miR-29a levels in skeletal muscle biopsies. The dashed lines indicate the intercept points corresponding to a $\Delta$ value of 1 , i.e., no change in HOMA-IR or miR-29a levels. miRNA levels were analyzed by qRT-PCR and normalized to miR-let-7. Results are shown as fold change of values after GHRT compared to values before GHRT. ${ }^{*} p<0.05,{ }^{* *} p<0.01$ (Student's $t$ test)

Moreover, we cannot rule out the possibility that miR-29a targets additional proteins of the ECM during GHRT. The downregulation of miR-29a could also impact the insulin response in skeletal muscle by targeting intracellular regulators of insulin signaling. Indeed, we identified PTEN as an endogenous target of miR-29a in human myotubes. PTEN is an inhibitor of insulin signaling and its upregulation following GHRT would expect to decrease the insulin response in muscle tissue [27]. However, most miR-29a targets are part of the ECM and affect the interaction of myofibers with their milieu. Indeed, overexpression or inhibition of miR-29a in human myotubes in vitro did not alter insulin-induced AKT phosphorylation demonstrating that investigating the role of miR29a for muscle insulin resistance will depend on reducing miR-29a levels in skeletal muscle in vivo.

We did not observe any correlation between miR-29a and insulin sensitivity in obese humans or mice with diet-induced obesity, indicating differences in mechanisms of insulin 


\section{a Human SM in GHRT}

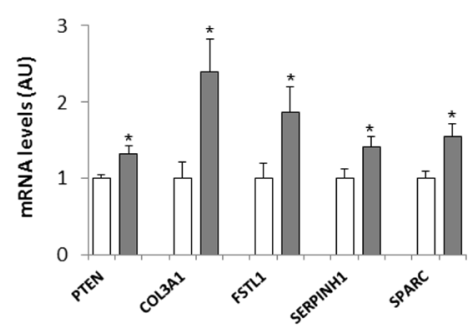

HOMA-IR decreased

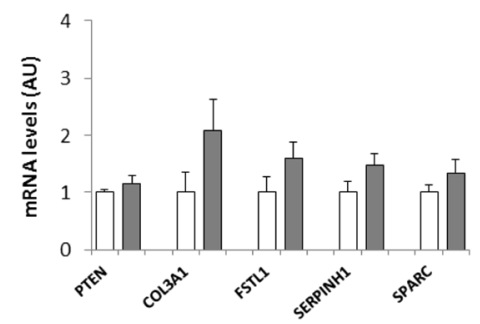

HOMA-IR increased

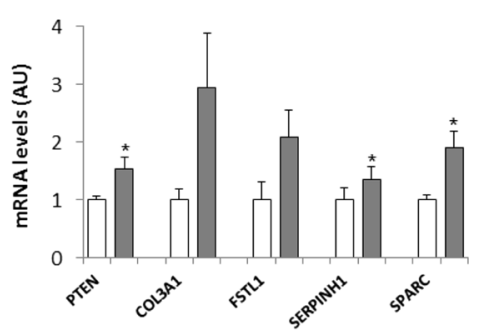

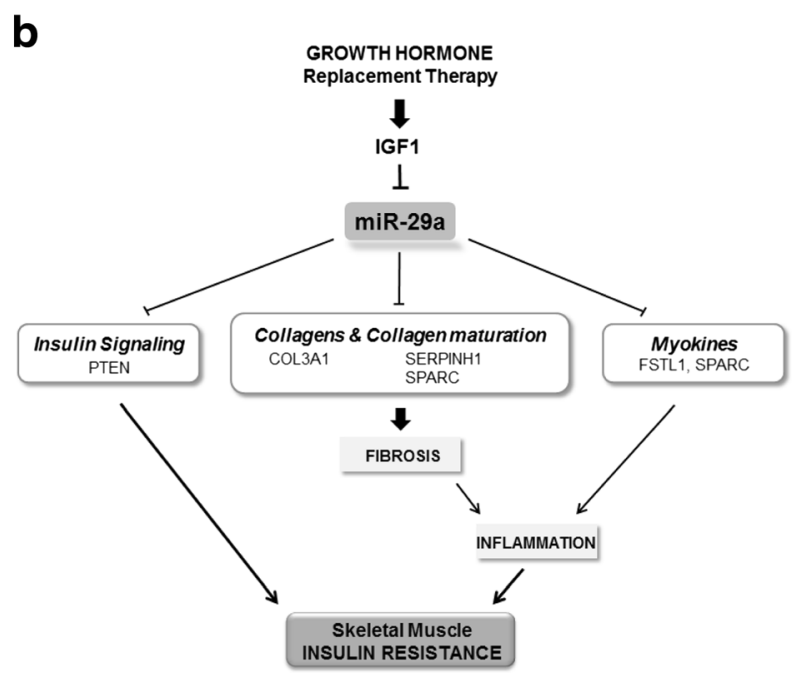

Fig. 5 Regulation of miR-29a targets in human skeletal muscle after GHRT. RNA was isolated from biopsies of the tibialis anterior muscle obtained from the GHD patients described in Fig. 4. a qRT-PCR analysis in skeletal muscle biopsies before (white) or after (gray) 4 months of GHRT, $n=9$, or separately analyzed for HOMA-IR decreased, $n=5$, and HOMA-IR increased groups, $n=4$. Results were normalized to $18 \mathrm{~S}$ RNA

resistance in skeletal muscle during GHRT and obesity. However, the average fasting insulin level in the prediabetic subjects $(0.141 \mathrm{nmol} / \mathrm{l})$ might not have been high enough to activate the IGF1 receptor and affect miR-29a expression. In this regard, it would be interesting to assess miR-29a regulation in skeletal muscle from insulin-resistant or diabetic subjects with a more pronounced hyperinsulinemia.

Recently, miR-29a was reported to be upregulated in skeletal muscle from insulin-resistant mice fed HFD [46]. We and other groups $[47,48]$ did not find such an induction. Overall, we exclude a relevant regulation of miR-29a in skeletal muscle of HFD mice not only by miRNA qRTPCR but also by the absence of any changes in the expression of miR-29a target genes. Potential explanations for the discrepant results include the different response of C57BL6 substrains to HFD [49] or differences in the HFD itself. Intriguingly, miR-29a was among the highest downregulated miRNAs in human skeletal muscle during a 3-h hyperinsulinemic-euglycemic clamp [50]. The average insulin concentration achieved during this clamp was slightly above $1 \mathrm{nM}$ and could have been sufficient to activate the IGF1 receptor in skeletal muscle. Due to technical and shown as arbitrary units (AU). Student's $t$ test was used to compare the two groups. ${ }^{*} p<0.05$. b Hypothetical model demonstrating the role of GHRT for ECM remodeling and its association with insulin resistance in skeletal muscle. Decreased miR-29a levels and upregulation of its targets during GHRT could contribute to this regulation

limitations with the glucose clamp technique, we used HOMA-IR as a surrogate to determine insulin sensitivity. However, the significantly higher triglycerides in subjects with increased HOMA-IR support a clinically meaningful separation of the metabolic state in our study.

Compared to patients with improved insulin sensitivity following GHRT, miR-29a levels decreased in patients with deteriorated insulin sensitivity by $30 \%$ (Fig. $4 \mathrm{a}$ ). The impact of such miRNA regulation that persists over long periods of time, e.g., months, in vivo has not been experimentally validated yet. However, regulation of less than twofold of miR-499 is associated with the induction of the type 1 muscle program in active compared to sedentary human subjects [51] and a $40 \%$ induction of miR-375 is associated with altered pancreatic beta cell proliferation in obese mice [52]. Therefore, our data provide strong support that regulation of miR-29a and its targets can contribute to muscle insulin resistance.

Patients with GHD are very heterogeneous in terms of comorbidities, and the efficacy of GHRT on metabolic parameters is highly variable. The identification of markers for adverse metabolic effects of GHRT is therefore of clinical importance. If future studies with larger sample size confirm our 
results, miR-29a and its targets could be valuable biomarkers for a decreased insulin response in skeletal muscle during GHRT and might, for example, help to identify other serum factors than IGF1 that allow to non-invasively monitor the metabolic response of skeletal muscle when GH is replaced. Preclinical animal models for GHRT as described in our study could be used to characterize in more detail the development of insulin resistance and changes in exercise-induced plasma levels of miR-29a-regulated myokines.

Acknowledgments We thank all the patients for their enthusiasm in participating in this study. We are thankful to Dr. Edlira Luca, Zurich, for the critical reading of the manuscript. This study was supported by the SNF grant PP00P3_128474 to J.K., by an unrestricted grant of the Swiss Society of Endocrinology and Diabetology (SGED) to J.K., by the Clinical Research Priority Program "small RNAs" of the University of Zurich, by the SNF grant No. 3200B0-100146/1 to E.C., and a grant from the Deutsche Forschungsgemeinschaft (DFG): KFO152 (TP2) to A.L. and M.B. GH was generously supplied by Pfizer Switzerland.

Conflict of interest The authors declare that they have no competing interests.

\section{References}

1. Carroll PV, Christ ER, Bengtsson BA, Carlsson L, Christiansen JS, Clemmons D, Hintz R, Ho K, Laron Z, Sizonenko P et al (1998) Growth hormone deficiency in adulthood and the effects of growth hormone replacement: a review. Growth Hormone Research Society Scientific Committee. J Clin Endocrinol Metab 83:382395

2. Moller N, Jorgensen JO (2009) Effects of growth hormone on glucose, lipid, and protein metabolism in human subjects. Endocr Rev 30:152-177

3. Ghanaat F, Tayek JA (2005) Growth hormone administration increases glucose production by preventing the expected decrease in glycogenolysis seen with fasting in healthy volunteers. Metabolism 54:604-609

4. Moller N, Jorgensen JO, Abildgard N, Orskov L, Schmitz O, Christiansen JS (1991) Effects of growth hormone on glucose metabolism. Horm Res 36(Suppl 1):32-35

5. Kargi AY, Merriam GR (2013) Diagnosis and treatment of growth hormone deficiency in adults. Nat Rev Endocrinol 9:335-345

6. Maison P, Griffin S, Nicoue-Beglah M, Haddad N, Balkau B, Chanson P (2004) Impact of growth hormone (GH) treatment on cardiovascular risk factors in GH-deficient adults: a metaanalysis of blinded, randomized, placebo-controlled trials. J Clin Endocrinol Metab 89:2192-2199

7. Claessen KM, Appelman-Dijkstra NM, Adoptie DM, Roelfsema F, Smit JW, Biermasz NR, Pereira AM (2013) Metabolic profile in growth hormone-deficient (GHD) adults after long-term recombinant human growth hormone (rhGH) therapy. J Clin Endocrinol Metab 98:352-361

8. Filipsson Nystrom H, Barbosa EJ, Nilsson AG, Norrman LL, Ragnarsson O, Johannsson G (2012) Discontinuing long-term GH replacement therapy - a randomized, placebo-controlled crossover trial in adult GH deficiency. J Clin Endocrinol Metab 97:3185-3195

9. Bramnert M, Segerlantz M, Laurila E, Daugaard JR, Manhem P, Groop L (2003) Growth hormone replacement therapy induces insulin resistance by activating the glucose-fatty acid cycle. J Clin Endocrinol Metab 88:1455-1463
10. Lu J, Getz G, Miska EA, Alvarez-Saavedra E, Lamb J, Peck D, Sweet-Cordero A, Ebert BL, Mak RH, Ferrando AA et al (2005) MicroRNA expression profiles classify human cancers. Nature 435: 834-838

11. Jordan SD, Kruger M, Willmes DM, Redemann N, Wunderlich FT, Bronneke HS, Merkwirth C, Kashkar H, Olkkonen VM, Bottger T et al (2011) Obesity-induced overexpression of miRNA-143 inhibits insulin-stimulated AKT activation and impairs glucose metabolism. Nat Cell Biol 13:434-446

12. Trajkovski M, Hausser J, Soutschek J, Bhat B, Akin A, Zavolan M, Heim MH, Stoffel M (2011) MicroRNAs 103 and 107 regulate insulin sensitivity. Nature 474:649-653

13. Trepp R, Fluck M, Stettler C, Boesch C, Ith M, Kreis R, Hoppeler H, Howald H, Schmid JP, Diem P et al (2008) Effect of GH on human skeletal muscle lipid metabolism in GH deficiency. Am J Physiol Endocrinol Metab 294:E1127-1134

14. Moreno-Navarrete JM, Martinez-Barricarte R, Catalan V, Sabater M, Gomez-Ambrosi J, Ortega FJ, Ricart W, Bluher M, Fruhbeck G, Rodriguez de Cordoba $\mathrm{S}$ et al (2010) Complement factor $\mathrm{H}$ is expressed in adipose tissue in association with insulin resistance. Diabetes 59:200-209

15. Stumvoll M, Van Haeften T, Fritsche A, Gerich J (2001) Oral glucose tolerance test indexes for insulin sensitivity and secretion based on various availabilities of sampling times. Diabetes Care 24:796-797

16. Masternak MM, Panici JA, Wang F, Wang Z, Spong A (2010) The effects of growth hormone $(\mathrm{GH})$ treatment on $\mathrm{GH}$ and insulin/IGF1 signaling in long-lived Ames dwarf mice. The journals of gerontology Series A, Biological sciences and medical sciences 65:24-30

17. Krutzfeldt J, Rajewsky N, Braich R, Rajeev KG, Tuschl T, Manoharan M, Stoffel M (2005) Silencing of microRNAs in vivo with 'antagomirs'. Nature 438:685-689

18. Sotiropoulos A, Ohanna M, Kedzia C, Menon RK, Kopchick JJ, Kelly PA, Pende M (2006) Growth hormone promotes skeletal muscle cell fusion independent of insulin-like growth factor 1 upregulation. Proc Natl Acad Sci U S A 103:7315-7320

19. Mavalli MD, DiGirolamo DJ, Fan Y, Riddle RC, Campbell KS, van Groen T, Frank SJ, Sperling MA, Esser KA, Bamman MM et al (2010) Distinct growth hormone receptor signaling modes regulate skeletal muscle development and insulin sensitivity in mice. J Clin Invest 120:4007-4020

20. Resmini E, Morte B, Sorianello E, Gallardo E, de Luna N, Illa I, Zorzano A, Bernal J, Webb SM (2011) Identification of novel GHregulated genes in $\mathrm{C} 2 \mathrm{C} 12$ cells. Horm Metab Res 43:919-930

21. Baudry A, Lamothe B, Bucchini D, Jami J, Montarras D, Pinset C, Joshi RL (2001) IGF-1 receptor as an alternative receptor for metabolic signaling in insulin receptor-deficient muscle cells. FEBS Lett 488:174-178

22. Donahue LR, Beamer WG (1993) Growth hormone deficiency in 'little' mice results in aberrant body composition, reduced insulinlike growth factor-I and insulin-like growth factor-binding protein-3 (IGFBP-3), but does not affect IGFBP-2, -1 or -4. J Endocrinol 136: 91-104

23. Hubmacher D, Apte SS (2013) The biology of the extracellular matrix: novel insights. Curr Opin Rheumatol 25:65-70

24. Lewis BP, Burge CB, Bartel DP (2005) Conserved seed pairing, often flanked by adenosines, indicates that thousands of human genes are microRNA targets. Cell 120:15-20

25. Tam CS, Covington JD, Bajpeyi S, Tchoukalova Y, Burk D, Johannsen DL, Zingaretti CM, Cinti S, Ravussin E (2014) Weight gain reveals dramatic increases in skeletal muscle extracellular matrix remodeling. J Clin Endocrinol Metab 99:1749-1757

26. Richardson DK, Kashyap S, Bajaj M, Cusi K, Mandarino SJ, Finlayson J, DeFronzo RA, Jenkinson CP, Mandarino LJ (2005) Lipid infusion decreases the expression of nuclear encoded 
mitochondrial genes and increases the expression of extracellular matrix genes in human skeletal muscle. J Biol Chem 280:10290 10297

27. Pal A, Barber TM, Van de Bunt M, Rudge SA, Zhang Q, Lachlan KL, Cooper NS, Linden H, Levy JC, Wakelam MJ et al (2012) PTEN mutations as a cause of constitutive insulin sensitivity and obesity. N Engl J Med 367:1002-1011

28. Yakar S, Rosen CJ, Beamer WG, Ackert-Bicknell CL, Wu Y, Liu JL, Ooi GT, Setser J, Frystyk J, Boisclair YR et al (2002) Circulating levels of IGF-1 directly regulate bone growth and density. J Clin Invest 110:771-781

29. Isgaard J, Nilsson A, Vikman K, Isaksson OG (1989) Growth hormone regulates the level of insulin-like growth factor-I mRNA in rat skeletal muscle. The Journal of endocrinology 120:107-112

30. Doessing S, Heinemeier KM, Holm L, Mackey AL, Schjerling P, Rennie M, Smith K, Reitelseder S, Kappelgaard AM, Rasmussen $\mathrm{MH}$ et al (2010) Growth hormone stimulates the collagen synthesis in human tendon and skeletal muscle without affecting myofibrillar protein synthesis. J Physiol 588:341-351

31. Berria R, Wang L, Richardson DK, Finlayson J, Belfort R, Pratipanawatr T, De Filippis EA, Kashyap S, Mandarino LJ (2006) Increased collagen content in insulin-resistant skeletal muscle. Am J Physiol Endocrinol Metab 290:E560-565

32. Kang L, Lantier L, Kennedy A, Bonner JS, Mayes WH, Bracy DP, Bookbinder LH, Hasty AH, Thompson CB, Wasserman DH (2013) Hyaluronan accumulates with high-fat feeding and contributes to insulin resistance. Diabetes 62:1888-1896

33. Kang L, Mayes WH, James FD, Bracy DP, Wasserman DH (2014) Matrix metalloproteinase 9 opposes diet-induced muscle insulin resistance in mice. Diabetologia 57:603-613

34. Kang L, Ayala JE, Lee-Young RS, Zhang Z, James FD, Neufer PD, Pozzi A, Zutter MM, Wasserman DH (2011) Diet-induced muscle insulin resistance is associated with extracellular matrix remodeling and interaction with integrin alpha2beta1 in mice. Diabetes 60:416426

35. de Fougerolles AR, Sprague AG, Nickerson-Nutter CL, Chi-Rosso G, Rennert PD, Gardner H, Gotwals PJ, Lobb RR, Koteliansky VE (2000) Regulation of inflammation by collagen-binding integrins alpha1beta1 and alpha2beta1 in models of hypersensitivity and arthritis. J Clin Invest 105:721-729

36. Kawasaki K, Ushioda R, Ito S, Ikeda K, Masago Y, Nagata K (2015) Deletion of the collagen-specific molecular chaperone Hsp47 causes endoplasmic reticulum stress-mediated apoptosis of hepatic stellate cells. J Biol Chem 290:3639-3646

37. Bradshaw AD (2009) The role of SPARC in extracellular matrix assembly. J Cell Commun Signal 3:239-246

38. Aoi W, Naito Y, Takagi T, Tanimura Y, Takanami Y, Kawai Y, Sakuma K, Hang LP, Mizushima K, Hirai Y et al (2013) A novel myokine, secreted protein acidic and rich in cysteine (SPARC), suppresses colon tumorigenesis via regular exercise. Gut 62:882889

39. Gorgens SW, Raschke S, Holven KB, Jensen J, Eckardt K, Eckel J (2013) Regulation of follistatin-like protein 1 expression and secretion in primary human skeletal muscle cells. Arch Physiol Biochem 119:75-80

40. Fan N, Sun H, Wang Y, Wang Y, Zhang L, Xia Z, Peng L, Hou Y, Shen W, Liu R et al. (2013) Follistatin-like 1: a potential mediator of inflammation in obesity. Mediators of inflammation 2013: 752519. DOI 10.1155/2013/752519

41. Kos K, Wong S, Tan B, Gummesson A, Jernas M, Franck N, Kerrigan D, Nystrom FH, Carlsson LM, Randeva HS et al (2009) Regulation of the fibrosis and angiogenesis promoter SPARC/ osteonectin in human adipose tissue by weight change, leptin, insulin, and glucose. Diabetes 58:1780-1788

42. Xu L, Ping F, Yin J, Xiao X, Xiang H, Ballantyne CM, Wu H, Li M (2013) Elevated plasma SPARC levels are associated with insulin resistance, dyslipidemia, and inflammation in gestational diabetes mellitus. PLoS One 8, e81615. doi:10.1371/journal.pone.0081615

43. Miyabe M, Ohashi K, Shibata R, Uemura Y, Ogura Y, Yuasa D, Kambara T, Kataoka Y, Yamamoto T, Matsuo K et al (2014) Muscle-derived follistatin-like 1 functions to reduce neointimal formation after vascular injury. Cardiovasc Res 103:111-120

44. Doessing S, Holm L, Heinemeier KM, Feldt-Rasmussen U, Schjerling P, Qvortrup K, Larsen JO, Nielsen RH, Flyvbjerg A, Kjaer M (2010) GH and IGF1 levels are positively associated with musculotendinous collagen expression: experiments in acromegalic and GH deficiency patients. Eur J Endocrinol 163:853-862

45. Chikani V, Ho KK (2014) Action of GH on skeletal muscle function: molecular and metabolic mechanisms. J Mol Endocrinol 52: R107-123

46. Yang WM, Jeong HJ, Park SY, Lee W (2014) Induction of miR-29a by saturated fatty acids impairs insulin signaling and glucose uptake through translational repression of IRS-1 in myocytes. FEBS Lett 588:2170-2176

47. Chen GQ, Lian WJ, Wang GM, Wang S, Yang YQ, Zhao ZW (2012) Altered microRNA expression in skeletal muscle results from high-fat diet-induced insulin resistance in mice. Mol Med Rep 5:1362-1368

48. Mohamed JS, Hajira A, Pardo PS, Boriek AM (2014) MicroRNA149 inhibits PARP-2 and promotes mitochondrial biogenesis via SIRT-1/PGC-1alpha network in skeletal muscle. Diabetes 63: 1546-1559

49. Harley IT, Giles DA, Pfluger PT, Burgess SL, Walters S, Hembree J, Raver C, Rewerts CL, Downey J, Flick LM et al (2013) Differential colonization with segmented filamentous bacteria and Lactobacillus murinus do not drive divergent development of dietinduced obesity in C57BL/6 mice. Molecular metabolism 2:171183

50. Granjon A, Gustin MP, Rieusset J, Lefai E, Meugnier E, Guller I, Cerutti C, Paultre C, Disse E, Rabasa-Lhoret R et al (2009) The microRNA signature in response to insulin reveals its implication in the transcriptional action of insulin in human skeletal muscle and the role of a sterol regulatory element-binding protein-1c/myocyte enhancer factor 2C pathway. Diabetes 58:2555-2564

51. Gan Z, Rumsey J, Hazen BC, Lai L, Leone TC, Vega RB, Xie H, Conley KE, Auwerx J, Smith SR et al (2013) Nuclear receptor/ microRNA circuitry links muscle fiber type to energy metabolism. J Clin Invest 123:2564-2575

52. Poy MN, Hausser J, Trajkovski M, Braun M, Collins S, Rorsman P, Zavolan M, Stoffel M (2009) miR-375 maintains normal pancreatic alpha- and beta-cell mass. Proc Natl Acad Sci U S A 106:58135818 